\title{
A Sensor based autonomous climbing vehicle for unstructured environments.
}

\author{
N.R.Bevan ${ }^{a}$ BEng(Hons), Prof.A.A.Collie ${ }^{b}$, \\ B.L.Luk ${ }^{b}$ PhD MSc BSc(Hons), T.S. White ${ }^{a}$ BEng(Hons).
}

aPortsmouth Technology Consultants (Portech) Ltd., Unit 12, Airport Service Road, Anchorage Park, Portsmouth, Hampshire, PO3 5RQ U.K.

${ }^{b}$ University of Portsmouth, Portech Mobile Robotics Laboratory, School of Systems Engineering, Anglesea Building, Anglesea Rd., Portsmouth, PO1 3DJ, U.K.

\begin{abstract}
This paper describes the latest developments to Robug IIs and in particular the modifications and additions to its sensor system and how this has helped to increase its autonomy in unstructured environments. The configuration of Robug IIs' local intelligence is described along with the system for control of each leg. The paper also describes Robug IIs' ability to perform automatic floor-to-wall transitions along with its alignment and positioning to the wall or obstacle by means of ultrasonic ranging. The final section of the paper describes the future enhancements being considered with a view to further increasing the autonomy.
\end{abstract}

\section{Introduction}

Apart from transporting components around the workplace mobile robots can also be used to carry tools to perform various remote operations. Such robots can provide a cost effective alternative for applications such as inspection or maintenance of large buildings, chemical plants or nuclear reactors etc. Such applications are either hazardous to human beings or involve erecting costly scaffolding. 
Robug IIs has been developed at the Portech Mobile Robotics Laboratory of the University of Portsmouth and is being used to address the problems of unstructured environments. Robug IIs is primarily controlled by a teleoperator control system consisting of a video display (displaying views from the on-board camera system) and computer interface for user interaction. The user controls the robot by use of high level commands which cause the robot to carry out coordinated motion subject to pre-programmed instructions.

The robot is able to cope with a relatively disordered environment but the control becomes more difficult when entering an unstructured environment where unforeseen obstacles are encountered. It is for this reason that we are decreasing the need for operator interaction and increasing the autonomy of Robug IIs.

Robug IIs is able to manoeuvre on horizontal and vertical surfaces. It also has the ability to perform autonomous floor-to-wall transitions to overcome obstacles such as walls and other large objects and has the intelligence to seek and verify foot holds.

This paper describes the sensor system developed for Robug IIs and describes how it uses this information to avoid obstacles and perform floor to wall transitions.

Its sensor system incorporates an ultrasonic ranging system controlled by a specially designed microcontroller board. The system is designed to aid with alignment and ranging during automatic movement. The sensor system also incorporates ;

(i) Attitude sensors

(ii) Vision

(iii) Tactile sensing

The paper will describe these systems in detail and will discuss their use in performing tasks in relatively unstructured environments. Other sensors in the system are potentiometers for recording leg positions and pressure transducers for determining leg forces. These parts of the system will also be described in some detail.

\section{Microcontroller configuration}

\subsection{Computer control system}

Control of Robug IIs is based on a hierarchial control system. At the top of the system hierarchy is a PC which serves as a supervisory computer. It performs path planning, on-line user interaction and the functions necessary for coordinated motion.

A master-slave network configuration via a RS232 serial link is used for communication between the supervisory computer and five individual microcontrollers see figure 1. The microcontroller card is based on the NEC 78310a microprocessor. Each card is dedicated to a specific part of the mechanical structure. Cards 1 to 4 are dedicated to control of an individual leg and the 5 th card is dedicated to external sensor control. 
All the microcontrollers listen to the network and each microcontroller has its own identification code, the board becomes active when it receives a board selection signal with its own identity code. The microcontroller then executes all the subsequent commands until a different microcontroller is selected. Upon receiving a command the microcontroller echoes the received command back to the supervisory computer to confirm the command. This arrangement simplifies the communication protocol and still achieves a high reliability of communication. Communication between the individual boards is carried out via the supervisory computer.

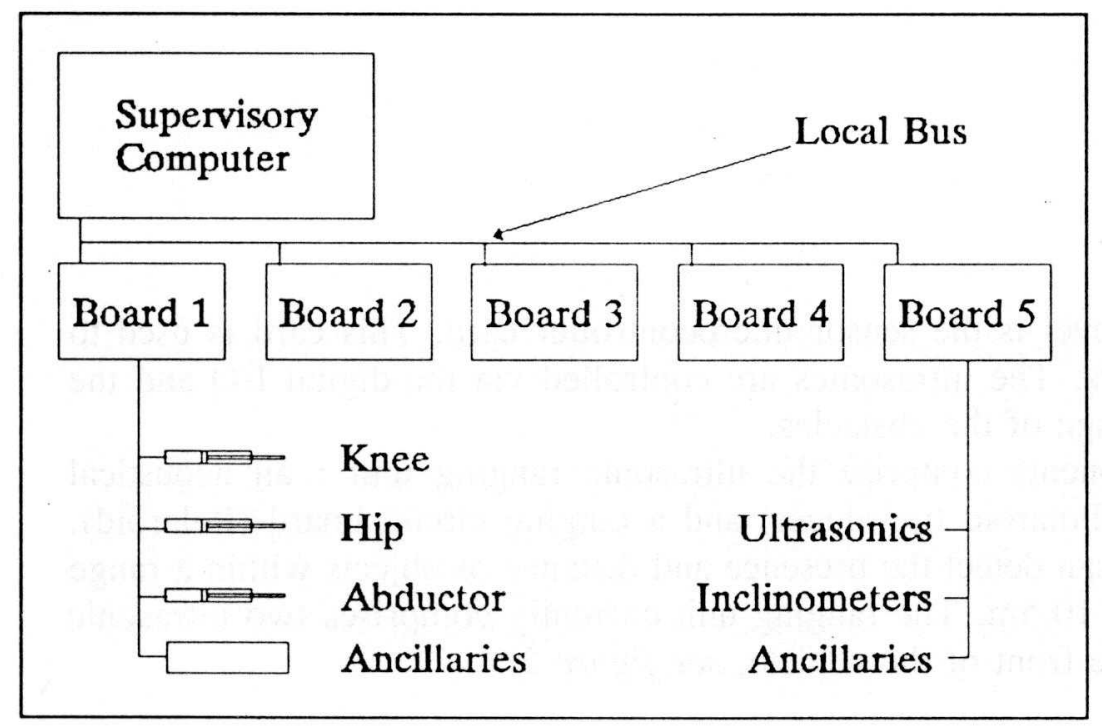

Figure 1. Configuration of hierarchial control structure.

\subsection{Leg control}

At the lower level are the individual limb controllers, each of which is a dedicated microcontroller board. The limb microcontroller controls all three joints of a single limb. To do this it uses the information from the potentiometers and pressure transducers to calculate leg positions and leg forces.

Each leg is controlled by nine electrically operated valves ( 3 for each cylinder). In order to provide the capability for Robug IIs to negotiate and climb over obstacles, each joint can be controlled in position control mode or compliant control mode. In the first mode the microcontroller uses position feedback to control the movement of the leg. This mode does not take into account the force feedback and hence is unable to detect obstacles in unstructured environments.

In the compliant control mode the individual microcontrollers monitor both position and force feedback. The demand pressure of the cylinders is calculated by multiplying the position error with a stiffness coefficient plus the force demand see equation 1. 
By adjusting the stiffness coefficient and the force demand, compliant control can be achieved. In this mode obstacles can be detected and the robot can feel for foot holds on the surface e.g. if the leg is obstructed the pressures will build.

$\Psi=\mathbf{K} \cdot \mathbf{e}+\mathbf{F}_{\mathrm{D}}$

$\boldsymbol{\Psi}$ is the demanded pressure, $\mathbf{e}$ is the position error, $\mathbf{K}$ is stiffness coefficient, $\mathbf{F}_{\mathrm{D}}$ is the force demanded

\subsection{Ultrasonic control}

Again at the lower level is the sensor microcontroller card. This card is used to control the ultrasonic sensors. The ultrasonics are controlled via the digital $\mathrm{I} / \mathrm{O}$ and the time stored relates to the range of the obstacles.

Two primary components comprise the ultrasonic ranging unit : an acoustical transducer (40mm, 50Khz Polaroid transducer) and a ranging circuit board (Polaroid). Together these components can detect the presence and distance of objects within a range of approximately $150 \mathrm{~mm}$ to $10.5 \mathrm{~m}$. The ranging unit currently comprises two ultrasonic transducers positioned on the front of the vehicle, see figure 2.

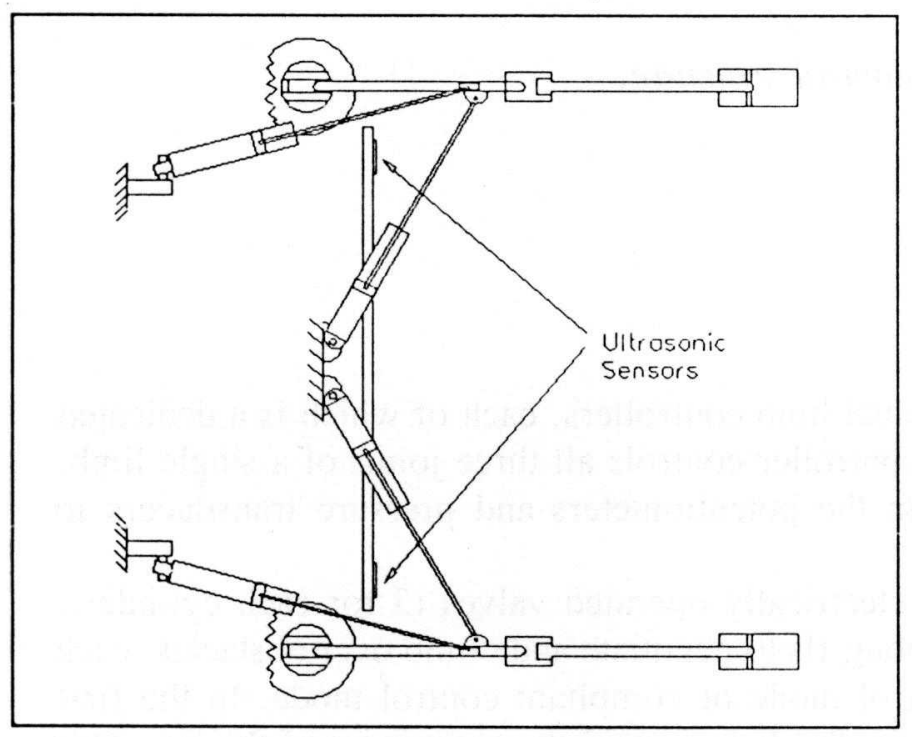

Figure 2. Front section of Robug IIs, showing position of Ultrasonic sensors. 
These two transducers are activated independently by the microcontroller card. Once the unit is activated the micro controller card starts a counter and the transducer emits a sound pulse. The emitted pulse is a $50 \mathrm{kHz}$ pulse lasting for about $1 / 2$ millisecond and consisting of sixteen pulses. The unit then waits to receive the echo returning from the object.

Upon receiving the echo the unit signals the microcontroller card with an echo received signal and the microcontroller stops the counter. This time is passed to the high level controller where it is used for alignment and ranging purposes.

\section{Floor-to-wall transition and obstacle avoidance.}

Whilst Robug IIs is approaching a wall or obstacle it is very easy for the teleoperator to see obstacles or walls in front of the vehicle but it is difficult for the teleoperator to judge the distance to that obstacle or wall using the on-board vision carried by Robug IIs.

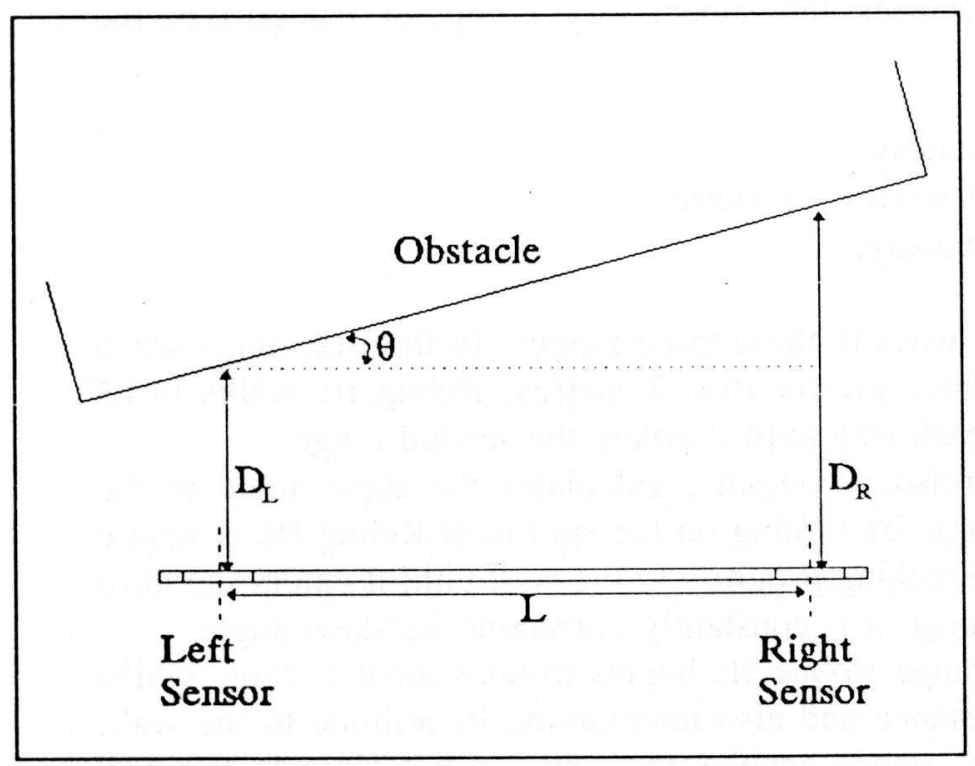

Figure 3. Configuration of ultrasonic sensors.

The information collected by the sensor board now becomes very useful. The ultrasonic sensors on Robug IIs are triggered independently by the supervisory computer via the serial link. The microcontroller card returns two independent times to the supervisory computer, one from the left and one from the right sensors. Figure 3 shows the configuration of the sensors on the front of Robug IIs. 
The high level software uses the information received from both the left and right sensors to calculate first of all the distance see equation 2 and secondly the skew angle of the obstacle to the robot, see equation 3.

\section{$D_{L}=\left(C * \Gamma_{L}\right) / 2$}

$D_{L}$ is the distance from the left sensor to the obstacle, $C$ is the speed of ultrasound in air and $\Gamma_{L}$ is the returned time for the left sensor from the sensor microcontroller. It can be seen that a similar calculation can be done for the right sensor.

$\theta=\tan ^{-1}\left[\left(D_{L}-D_{R}\right) / L\right]$

$\theta$ is the skew angle of the robot to the obstacle/wall, $D_{L}$ is the distance from the left sensor to the obstacle/wall, $D_{K}$ is the distance from the right sensor to the obstacle/wall, and $L$ is the distance between the two sensors.

After calculating the two distances the supervisory computer categorises the distances into three levels:

(i) Obstacles/Walls over 2 metres away.

(ii) Obstacles/Walls in the range 2 metres - 1 metre.

(iii) Obstacles/Walls under 1 metre away.

Robug IIs behaves in different ways in these three ranges. In the first range when an obstacle/wall is detected at a distance greater than 2 metres, Robug IIs walks in its normal way, taking full strides with each step until it enters the second range.

In the second range the supervisory computer calculates the skew angle to the obstacle/wall it then alters its skew angle by turning on the spot until Robug IIs is square to the obstacle/wall. It then carries on making progress to the wall until it enters the third range. Whilst approaching the third range it is constantly correcting its skew angle.

Once it has entered the third range Robug IIs begins to take smaller steps, whilst gradually increasing the width of its stance and also maintaining its attitude to the wall. This action of increasing the width of stance enables the robot to get close to the wall without its feet hitting the wall. Robug IIs carries on moving closer to the obstacle/wall until it reaches a distance of about $1 / 2$ metre. At this point Robug IIs is ready to perform a floor to wall transition to manoeuvre over the obstacle or climb onto the wall.

The spine of Robug IIs can be controlled to assist with a floor-to-wall transition. Once the robot is in position to carry out the floor-to-wall manoeuvre it begins by bending the spine so that the front section is at approximately $45^{\circ}$ to the wall. Along with the movement of the legs this allows the front section of the robot to make the transition to the wall.

If however the wall is too far away for the front feet to make the transition the spine cylinder can be controlled and bend to a certain angle (specified by teleoperator interaction) to enable the front feet to make contact. 
After the robot has begun its floor-to-wall transition it uses its attitude sensor to keep it in a vertical orientation during the transition. The attitude sensor used on Robug IIs is an Accustar" electronic clinometer. The sensor is a capacitance based device and has no moving parts, it has a range of $\pm 60^{\circ}$ and a resolution of $0.001^{\circ}$. If Robug IIs begins to veer to the left or the right the Robug IIs supervisory computer adjusts the step length of the legs to compensate for this. The attitude sensor is also used to keep Robug IIs at a fixed angle on the wall, again adjusting step lengths etc. Figure 4. shows Robug IIs performing a floor to wall transition at the FRED test facility [1].

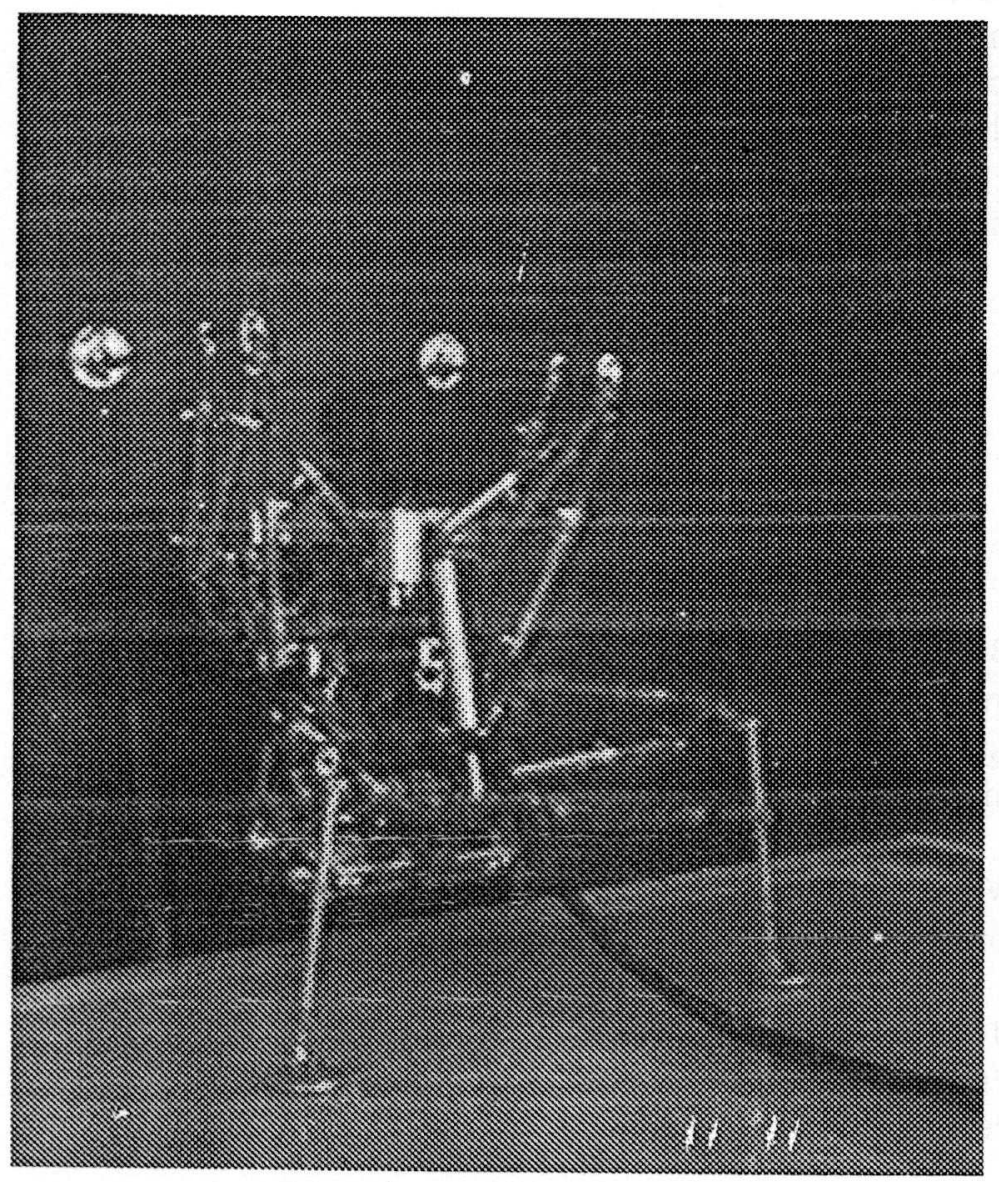

Figure 4. Robug IIs performing a floor to wall transition on FRED 


\section{Conclusions}

Robug IIs has been fully tested both at the Portech Mobile Robotics laboratory at the University of Portsmouth and at the FRED test facility on a full scale reactor mock-up. Its performance has been compared with the performance of the Nero [2] vehicles developed by Portech for Nuclear Electric Plc which successfully completed its part of a 12 month inspection programme at the Trawsfynydd Nuclear Power Station [3-5]. Robug IIs has the advantages of floor to wall transfer over the Nero robot and was found to have a comparable ability once situated on the vessel. Following these trials further modifications are being carried out to further improve the autonomy of the system. Some of the enhancements being considered follow ;

Robug IIs is able to perform the alignment to the wall and the subsequent floor-to wall transition but as yet is unable to perform the transition from wall-to-floor. It is not felt that a pair of ultrasonic sensors are required merely a single sensor on the rear of the robot to give us a distance to the floor. The alignment with the floor can be achieved using the attitude sensor found on Robug IIs.

Enhancement of the vision system would also lead to increased autonomy. Especially in the fields of automatic path planning/obstacle avoidance.

Whilst work on Robug IIs is being carried out another robot development which has recently begun is another version of the Robug robot, Robug III. This project is a two year Teleman scheme funded by the Commission of the European Communities.

\section{References}

1 FRED - Facility for Reactor Engineering Development, Nuclear Electric Plc.

2 T.S.White, A.A.Collie, J.Billingsley, B.L.Luk, and D.A.Moseley, "A Robot climbing vehicle and its Man-Machine interface for use in the nuclear industry", The 8th International Symposium on Automation and Robotics in Construction, 3-5 June 1991, Stuttgart, Germany, pp 851-859.

3 A.A.Collie, B.L.Luk, T.S.White and N.R.Bevan, "Experience of climbing robots on a nuclear reactor", IBC conference on Remote Technology for the Nuclear Ind.

4 A.Curry, M.S.Burrows and R.P.F.Clayton, "Nuclear reactor pressure, vessel inspection climbing vehicles/robots", IBC conference on Remote Technology for the Nuclear Industry, 2-3 December 1991, London.

5 M.S.Burrows, A.A.Collie and A.Curry, "Inspection of a nuclear reactor pressure vessel by vacuum attached and magnetically attached mobile robots", 4th International Symposium on Offshore, Robotics and Artificial Intelligence Telerobotics in Hostile Environments, 11-12 December 1991, Marseille, France. 